\title{
AGENCIAMIENTO DE LOS CAMBIOS LEGALES EN LA FORMACIÓN DE LOS MAESTROS NORMALISTAS DE SANTIAGO DE CALI (2004-2016) ${ }^{1}$
}

\author{
Laura María Pineda Villany ${ }^{2}$ \\ Claudia del Pilar Vélez De la Calle ${ }^{3}$
}

\begin{abstract}
RESUMEN/ ABSTRACT
En este artículo se pretende, en primer lugar, problematizar el asunto referido a la ausencia de un marco legal adecuado para las Escuelas Normales en Colombia que permita dar respuesta efectiva a las funciones asignadas por la ley para la formación de maestras y maestros. En segundo lugar, se busca caracterizar algunos de los aspectos determinantes de la vida cotidiana de las Escuelas Normales. Finalmente, se proponen algunos agenciamientos de la formación del maestro, de orden pedagógico, curricular y organizativo, a partir del agenciamiento de la formación del maestro en el contexto de las Escuelas Normales Superiores de Santiago de Cali (Colombia).
\end{abstract}

Palabras clave: agenciamiento; cambios legales; escuela normal; formación del maestro; currículum.

In this article, it is intended, first, to problematize the matter referred to the absence of an adequate legal framework for the Normal Schools in Colombia that allows an effective response to the functions assigned by law for the training of teachers. Secondly, it seeks to characterize some of the determining aspects of the daily life of the Normal Schools. Finally, some agency for teacher training, of pedagogical, curricular and organizational order, are proposed, starting with the formation of the teacher in the context of the Superior Normal Schools of Santiago de Cali (Colombia).

Keywords: Agency, legal changes, normal school, teacher training, curriculum.

\footnotetext{
${ }^{1}$ Este artículo es resultado de una investigación doctoral realizada en el Doctorado en Educación de la Universidad

${ }^{2}$ Lic. en Educación; Mg. en Educación, Pontificia Universidad Javeriana; Candidata a Doctora en Educación; Docente, Normal Superior Santiago de Cali; Docente Facultad de Educación, Universidad San Buenaventura de Cali; 1mpvilla@usbcali.edu.co

${ }^{3}$ Lic. en Educación; Mg. en Educación y Desarrollo Social; Doctora en Teoría de la Educación y Pedagogía Social, Universidad Nacional de Educación a Distancia, UNED, España; Directora del Doctorado en Educación de la Universidad San Buenaventura de Cali. Email: cpvelez@usbcali.edu.co
} 


\section{INTRODUCCIÓN: ESTADO DE LA CUESTIÓN}

Históricamente las Escuelas Normales Superiores en Colombia se han trasformado y desarrollado a la luz de políticas, normas y planes educativos que han señalado el valor social y político de la formación de las/os docentes. Lo afirmado se sustenta en el artículo 68 de la Constitución Política de 1991: “(...) la enseñanza estará a cargo de personas de reconocida idoneidad ética y pedagógica"; y en lo precisado en el artículo 4 de la Ley 115 de 1994, en el cual se considera que corresponde

[al] Estado, a la sociedad y a la familia velar por la calidad de la educación y promover el acceso al servicio público educativo, y es responsabilidad de la Nación y de las entidades territoriales, garantizar su cubrimiento. El Estado deberá atender en forma permanente los factores que favorecen la calidad y el mejoramiento de la educación; especialmente velará por la cualificación y formación de los educadores, la promoción docente, los recursos y métodos educativos, la innovación e investigación educativa, la orientación educativa y profesional, la inspección y evaluación del proceso educativo. (Congreso de la República, 1994)

Consecuente con lo señalado, el Ministerio de Educación Nacional propone el Sistema Colombiano de Formación de Educadores y Lineamientos de Política (M.E.N, 2013) como un marco de referencia que ofrece directrices que orientan la formación de las/os docentes del país. En este se propone el desarrollo de estrategias que viabilicen la formación y fortalecimiento de competencias básicas y profesionales en las/los docentes en formación y en quienes se encuentran en servicio, con el propósito de aportar herramientas pedagógicas y didácticas que contribuyan a la transformación del quehacer pedagógico y la/s práctica/s pedagógica/s; y permitan reconocer contextos, y responder pedagógicamente a las diferentes capacidades y necesidades de las/os estudiantes en procura del aprendizaje y desarrollo de sus competencias para la vida. Para tal fin se definen tres ejes transversales que articulan y potencian la formación de el/la maestro/a colombiano/a. 
El primer eje es la pedagogía como saber esencial, teórico y práctico, que da sentido e identidad a la formación de educadoras/es. De acuerdo con esto, la formación pedagógica es un elemento central a lo largo de la formación de las/os maestras/os, en tanto

la apropiación, desarrollo y constitución disciplinar del conocimiento pedagógico desde la perspectiva de la comprensión de los procesos de enseñanza y aprendizaje, los ambientes y el contexto para el aprendizaje, la didáctica, el currículo, la evaluación y la escuela como lugar de socialización entre otros temas asociados a la educación[que dan sustento a la práctica pedagógica del docente]. (M.E.N, 2013, p. 64)

El segundo eje es la investigación como dinamizadora de la producción y divulgación del saber pedagógico, así como de la reflexión sobre las prácticas de las/os docentes. Este eje tiene la intención de promover la actitud reflexiva, esto es, la capacidad de asombro, indagación, creación, interés y deseo por conocer y explicar la realidad, de manera tal que las/os maestras/os en formación y en servicio avancen desde la acumulación de contenidos hacia la comprensión de conceptos y procesos para su ejercicio presente y futuro.

El tercer eje es la evaluación como actividad de aprendizaje y conocimiento a través de la cual se obtiene información para cualificar las prácticas de formación de docentes y las prácticas mismas de la/el docente.

En el documento Sistema Colombiano de Formación de Educadores y Lineamientos de Política, se expresa que las Escuelas Normales Superiores son instituciones formadoras que tienen el reto de formar integral y profesionalmente a la/el maestra/o normalista, permitiendo un acercamiento significativo y profundo a la pedagogía, a la profesión y al rol docente. Para esto, de conformidad a lo estipulado por el Ministerio de Educación Nacional, se requiere:

Formar a los educadores en un ámbito de interpretación y comprensión de la realidad educativa nacional y regional, además de estudiar las implicaciones sociales, culturales, cognitivas, personales y disciplinares de la educación desde sus distintos niveles y desarrollos. Esto es, incursionar en un proceso de formación integral, permanente y de mejoramiento continuo que permita al educador actuar ante las necesidades de la educación. La formación como educador está dirigida a aprender a enseñar, a posibilitar el aprendizaje de diversos conocimientos, competencias, 
contenidos conceptuales, actitudinales y procedimentales, con el fin de crear posibilidades vitales para la constitución de los sujetos sociales a través de la educación. (M.E.N, 2013, p. 72)

En el artículo 112 de la Ley General de Educación, en su parágrafo se comenta respecto de la estructuración de las Escuelas Normales Superiores, señalando que estas:

Debidamente reestructuradas y aprobadas, están autorizadas para formar educadores en el nivel de Educación Preescolar y en el nivel de Educación Básica el ciclo Primaria. Operarán como unidades de apoyo académico para la formación inicial de docentes y, podrán ofrecer formación complementaria que conduzca al otorgamiento del título de normalista superior

En el Programa de Formación Complementaria, las prácticas pedagógicas se proponen como el quehacer de la maestra y del maestro en el contexto de la educación preescolar y de básica primaria y se las concibe desde una relación dialógica entre teoría y práctica que se logra a través de la reflexión crítica sobre el hacer y quehacer de la práctica. Esta reflexión está orientada a la producción y renovación del conocimiento teórico, es decir, es una praxis social intencionada, donde intervienen las apreciaciones y acciones del maestro en los procesos de enseñanza y aprendizaje. A tenor de lo mencionado, se advierte como estas prácticas reflejan los paradigmas que configuran el pensamiento y la acción de los docentes.

Así las cosas, la naturaleza y razón de ser de la Escuela Normal Superior en Colombia es la de "formar maestros/as de excelente calidad humana, ética, pedagógica, investigativa, para liderar procesos educativos innovadores en los niveles de Educación Preescolar y en Educación Básica Primaria; potenciar el desarrollo humano de las nuevas generaciones y contribuir a la transformación de su entorno como agentes sociales de cambio.” (Proyecto Educativo Normalista [PEN], año 2012, p. 15).

La práctica pedagógica se constituye en elemento fundamental, en el que la/el normalista en formación se mueve entre dos conocimientos: el formal (teórico, enfoques, estrategias y técnicas) y el experiencial (aplicación de estrategias pedagógicas, vivencias, solución de conflictos). De esta forma, la práctica pedagógica se configura como un proceso dinamizador que 
favorece el conocimiento de las/os estudiantes y el vínculo entre la academia y la realidad social; es un espacio de reflexión, de análisis sobre los hechos que acontecen en el contexto educativo.

Ahora bien, esta práctica pedagógica de carácter investigativa constituye el proceso de apropiación del papel de la/el docente en acción. En este sentido, se asume que la/el maestra/o en formación es quien logra su formación y autoformación, puesto que se parte del presupuesto de que ganar experiencias es posible en la medida en que cada cual viva intensamente el sentido del ejercicio de la docencia y esto se logra a través de la "praxis pedagógica" entendida como la "actualización de la proximidad, de la experiencia del ser, del construir al otro como persona, como fin de mi acción y no como medio" (Peña, 1996 , p. 17). En ella y dentro de ella, la/el maestra/o en formación tiene la posibilidad de aprender y desaprender basándose en la experiencia individual y colectiva, legitimando el uso de herramientas que le permitan integrar, argumentar, proponer, demostrar y resolver los fenómenos educativos propios de su cotidianidad a la vez que plantear nuevas formas de relación con la realidad existente. Esta "praxis pedagógica" debe proporcionar una formación integral, para lo cual se hace necesario que la Escuela Normal Superior brinde los espacios que posibiliten los siguientes requerimientos:

- La articulación de la Educación Media y el Programa de Formación Complementaria de tal manera que la intencionalidad educativa esté consolidada con un enfoque hacia la formación de la/el docente, comprometiendo una visión del ser humano, la sociedad y la educación.

- La integración de la cultura académica y disciplinaria a la cultura de la cotidianidad, en donde se tenga en cuenta los diferentes contextos: aula, institución y comunidad.

- El trabajo interdisciplinario del colectivo docente, donde cada cual, desde su campo de formación, aporta los elementos conceptuales y participa en su desarrollo de tal manera que se logre la integración de las disciplinas alrededor de un mismo tema, proyecto o temática.

- La articulación de saberes, el fomento de las relaciones pedagógicas, la reflexión permanente de la comunidad académica, la identificación de estrategias pedagógicas que posibiliten el florecimiento de la investigación pedagógica. 
- La respuesta a los intereses y necesidades de los diferentes grupos poblacionales, reconociendo sus especificidades, a fin de brindar oportunidades de acceder, permanecer y promocionarse en condiciones apropiadas de calidad, pertinencia y eficiencia.

\section{EL PROBLEMA DE LA FORMACIÓN NORMALISTA}

La Ley General de Educación, Ley 115 de 1994, artículo 107, es un hito relevante que exigió a las Escuelas Normales Superiores en Colombia, renovación y cambio. Es así como surge el Programa de Formación Complementaria (PFC) con el objetivo de formar en la/el maestra/o normalista competencias profesionales sobre la base de la incorporación y producción de conocimientos y prácticas fundamentadas en el conocimiento científico, cultural y pedagógico, en enfoques y modelos pedagógicos, y en la lectura del contexto. La propuesta se realiza a la luz de los principios de Educabilidad - Enseñabilidad - Pedagogía y Contexto y de los Ejes de Formación- Evaluación - Investigación y Práctica Pedagógica.

En el PFC la Práctica Pedagógica se propone a la luz de las teorías y discursos que circulan en el ámbito escolar y en los temas de enseñanza obligatoria de Educación Preescolar y Básica Primaria. "Su razón de ser es "hacer visible lo cotidiano", como dirían Carr \& Kemmis (1988); se impone el examen crítico de la práctica por los propios prácticos, con intencionalidad emancipadora, con voluntad cooperativa con proposición dialógica.” (Ríos, 2004, p. 153)

Esta concepción de "práctica pedagógica" enmarca el perfil de una/un maestra/o que piensa en los problemas del contexto, capaz de considerar y evaluar su práctica día a día, de reflexionar sobre las teorías y los discursos de la educación, la pedagogía, la didáctica y el currículo, para proponer otras formas, otras maneras de pensar y de actuar, a la luz de la experiencia vivida; una/un maestra/o que se aproxima a constructos teóricos que conciben el pensamiento mismo como un producto social y cultural, en donde la investigación se muestra como estrategia y medio válido que hace posible comprender y apropiar la práctica pedagógica como un acto culturalmente construido que se da en la interacción cotidiana. 
Sin embargo, lo aquí expuesto, en la cotidianidad de las Escuelas Normales Superiores, se muestra extraño, contradictorio, diluido en el día a día, a razón del marco normativo que las reglamenta, que prescribe para ellas una doble condición: ser Escuelas Normales Superiores que ofertan un Programa de Formación Complementaria que forma profesionales para el ejercicio de la docencia, al que se le exige organización académica y administrativa propia de la educación superior, y ser Institución Educativa que oferta Educación Preescolar, Básica y Media.

La investigación de la cual da cuenta este artículo buscó hacer explícita la tensión que se genera a razón del horizonte normativo que regula el funcionamiento de la Institución Educativa y de la Escuela Normal Superior. Esta tensión se abordó desde la metáfora del "cuerpo ajeno", con la intencionalidad de aproximarse al asunto de la formación desde un lugar que se denominó “agenciamiento", tomando el concepto de Deleuze y Guattari (1999). El problema se planteó en los siguientes términos: ¿qué compresiones de los cambios legales vividos en el periodo de 20042016 y de su agenciamiento en la formación de la/el maestra/o normalista tienen las Escuelas Normales Superiores de Santiago de Cali?

La investigación tuvo por objetivo general interpretar las compresiones que las Escuelas Normales Superiores de Santiago de Cali tienen de los cambios legales vividos en el periodo de estudio establecido en la investigación (2004-2016) y de su agenciamiento en la formación del maestro normalista. Los objetivos específicos, por su parte, fueron recocer los marcos normativos y los cambios dados a razón de ellos; develar la comprensión que de los marcos normativos y de los cambios dados a razón de ellos tienen las/os formadoras/es de formadoras/es; y determinar las tensiones y tendencias en la formación de la/el maestro normalista que surgen de los cambios de las Escuelas Normales Superiores de Santiago de Cali.

El problema del agenciamiento de los cambios legales en la formación de las/os maestras/os normalistas de Santiago de Cali se abordó desde un enfoque cualitativo, en perspectiva hermenéutica, mediante un estudio de caso y triangulación de fuentes. Se asumió como el estudio de un todo integrado.

La investigación asumió, también, la idea de que, en lo cualitativo, el conocimiento es un producto social, atravesado e influenciado por los valores, percepciones y significados de quienes lo construyen a través de la reflexión sobre la acción de las personas y comunidades, y el 
reconocimiento de la realidad intersubjetiva. En la hermenéutica, quien investiga busca comprender e interpretar una realidad en un contexto concreto, además de interpretar los motivos internos de la acción humana. La/el hermeneuta pretende comunicar, traducir, interpretar, comprender, dar sentido y significar los mensajes no evidentes en los textos y en los contextos (historia, cultura, política, filosofía, sociología, educación, etc.).

La hermenéutica cuestiona la posición y la homogeneidad de quien interpreta, posee una concepción del conocimiento que cuestiona la conceptualización del otro como sujeto a ser comprendido y cuestiona la comprensión del sujeto mismo que comprende, siendo necesario descentrar su tradición y moverse hacia otra, lo que significa situarse en "otro lugar de enunciación". Mignolo denomina a esto el "pensamiento fronterizo" o "pensamiento otro" (2007b). El fundamento metódico de la hermenéutica no es la explicación, sino la comprensión. Comprender es el acto a través del cual se capta el mundo exteriorizado, es posibilidad epistémica y metódica que posibilita dar cuenta del estado de las realidades.

La hermenéutica se halla en instancias de cruces, bisagras y límites, desdibujando la linealidad de la tradición, y se enfrenta a lugares diversos, variados, plurales, múltiples. Se ubica en la epistemología de frontera, en el pensamiento otro, decolonial, que insta a un giro dado por la relocalización del locus de enunciación que se muda a aquellos lugares que han sido enmudecidos. Exige un acto interpretativo allí donde no hay ni homogeneidad ni una línea de continuidad con la tradición legada; se trata de tradiciones-culturas-horizontes en donde el sujeto es hacedor de la explotación, la dominación y la invisibilización de la otredad. En el caso de las Escuelas Normales, las normas colonizan su pensamiento y actuación pedagógica.

El estudio de casos busca comprender las dinámicas presentes en contextos singulares de las Escuelas Normales Superiores. A través de él se puede registrar y describir el comportamiento de las personas involucradas en el fenómeno-objeto de estudio: "Permite ir más allá de la experiencia descrita por los actores" (Walker \& Stuart, 1983); posibilita comprender en profundidad.

La investigación que aquí se presenta contribuye al campo de estudio sobre la comprensión del fenómeno educativo de las Escuelas Normales Superiores y su sostenibilidad institucional, lo que hace evidente el aporte al campo del pensamiento pedagógico, provocando 
posibilidades epistémicas que se sitúan en el ámbito de comprender el agenciamiento de la formación en las Escuelas Normales Superiores a partir de las normas que ordenan, prescriben e incorporan realidades, productoras estas de otra/a realidad/es: "pensamiento otro". Normas que desconocen el quehacer formativo de las/os docentes, que silencian la voz de los actores, que acallan el sentir y el pensar de el/la maestro/a formador/a en relación con la formación de la/el maestra/o normalista.

Las otras realidades, "pensamiento otro", se acompañan de la figura del agenciamiento que posibilitó un despliegue del pensamiento, un aporte y respuesta a una irregularidad en un cuerpo de conocimiento. Es ahí donde descansa la apuesta critica de esta investigación que entra en diálogo con los estudios culturales y el pensamiento pedagógico y que invita a una lectura crítica de la realidad de la formación de la/el maestro normalista, sobre lo normativo. Es una apuesta distinta acerca de cómo comprender el quehacer formativo de los maestros en el contexto de las Escuelas Normales Superiores de Santiago de Cali.

\section{DESARROLLO}

Un agenciamiento es precisamente ese aumento de dimensiones en una multiplicidad que cambia necesariamente de naturaleza a medida que aumenta sus conexiones.

Deleuze y Guattari (2004)

Es relevante precisar que el agenciamiento de las Normales Superiores, y en eso radica su importancia, rizomáticamente interconecta la vida y la/s voz/voces de los actores implicados ${ }^{4}$ (directivo docente, formador/a de formadores/as, maestra/o en formación); sus reflexiones y pensamientos en un escenario normativo que regula las Escuelas Normales Superiores y los cambios dispuestos en ellas; la organización escolar para darle sentido a la formación en coherencia con lo dispuesto por el Estado; y el presente y futuro inmediato de la institución

\footnotetext{
${ }^{4}$ En la investigación, la expresión "actores" se utiliza para referirse a directivos docentes, docentes y estudiantes normalistas.
} 
normalista, dada la inminente restructuración de ellas considerada en las "Políticas y Sistema Colombiano de Formación y Desarrollo Profesional Docente” vigente (MEN, s.f.).

Ahora bien, con el propósito de afincar esta propuesta en torno al agenciamiento de la formación del maestro normalista, resulta adecuado y pertinente conceptualizar primero lo que aquí se comprenderá por agenciamiento. Para tal fin, resulta relevante, la obra Mil mesetas (Deleuze \& Guattari, 2004).

Hay que advertir que la palabra agenciamiento aparece en Mil mesetas aproximadamente 552 veces. Dicha cifra determina, por un lado, la relevancia de esta en el pensamiento de estos autores contemporáneos y, por otro, la riqueza y multiplicidad que trae consigo, y su incidencia en toda micropolítica del entramado social, como se argumentará a continuación.

Así las cosas, lo que se pretende es hacer pedagogía del concepto de agenciamiento en correspondencia con la formación de la/el maestro normalista en las Escuelas Normales Superiores de Santiago de Cali. Esta pedagogía del concepto de agenciamiento, debe, necesariamente, "analizar las condiciones de creación como factores de momentos que permanecen singulares" (Deleuze y Guattari, 2013, p. 18) en la trama de la Normal Superior, tanto desde el horizonte ontológico, gnoseológico, antropológico, como normativo, ya que, por ejemplo, las normas son expresión de un acto de carácter ilocutorio que "se explica a su vez por agenciamientos colectivos de enunciación, por actos jurídicos, equivalentes de actos jurídicos, que distribuyen los procesos de subjetivación" (Deleuze y Guattari, 2004, p. 84), como se ha evidenciado en este escrito.

Este agenciamiento, comprendido en el seno de este escrito, opera como un rizoma que interconecta y aglutina cada uno de los eslabones existentes en la Normal Superior, tales como maestras/os, directivos docentes, estudiantes, normas y el contexto vital y territorial en el que estos eslabones descansan. Según Deleuze y Guattari, "nosotros no conocemos ni la cientificidad ni la ideología, solo conocemos agenciamientos" (2004, p. 26-27), los cuales, en su multiplicidad, actúan entre sí, intervinculados reticularmente, procedentes de un mismo contexto vital configurado por una territorialidad y un conglomerado social. 
Sumado a esto hay que mencionar, por un lado, que la primera condición de agenciamiento es el territorio, en otras palabras, el contexto territorial de Santiago de Cali. Para Deleuze y Guattari, "el propio territorio es un lugar de paso. El territorio es el primer agenciamiento, la primera cosa que hace agenciamiento, el agenciamiento es en primer lugar territorial” (2004, p. 328). A lo que, más adelante, agregan los autores:

El territorio crea el agenciamiento. El territorio excede a la vez el organismo y el medio, y la relación entre ambos; por eso el agenciamiento va más allá también del simple comportamiento (de ahí la importancia de la distinción relativa entre animales de territorio y animales de medio). (2004, p. 513)

De otro lado, está el pensar la formación de maestras/os en las instituciones educativas Normal Superior desde un agenciamiento maquínico, lo cual implica reconocer una nueva producción de enunciados acerca de la formación; de ahí, la vigencia de esta propuesta, toda vez que se necesita del agenciamiento para que:

La unidad de composición englobada en un estrato, las relaciones entre tal estrato y los otros, la relación entre estos estratos y el plan de consistencia, estén organizadas y no sean cualesquiera. Bajo todos los puntos de vista, los agenciamientos maquínicos efectúan la máquina abstracta tal como está desarrollada en el plan de consistencia, o englobada en un estrato. (Deleuze y Guattari, 2004, p. 76)

Comprender e interpretar estas instituciones educativas en clave de agenciamiento resignifica los procesos formativos configurados en su interior, pues comienza por reconocer a los actores, sus relaciones, sus procesos de subjetivación, las relaciones de poder y de significado que se configuran, sus matices y consecuencias, producto de la unidad y composición englobada en el estrato Normal Superior. Cabe aquí recurrir al concepto de "estratos" que proponen Deleuze y Guattari (2004):

Los estratos son fenómenos de espesamiento en el Cuerpo de la Tierra, a la vez moleculares y molares: acumulaciones, coagulaciones, sedimentaciones, plegamientos. Son Cinturas, Pinzas o Articulaciones. Sumaria y tradicionalmente se distinguen tres grandes estratos: físico-químico, orgánico, antropomórfico (o 
aloplástico). Cada estrato, o articulación, se compone de medios codificados, sustancias formadas. Formas y sustancias, códigos y medios no son realmente distintos. Son las componentes abstractas de toda articulación. (p. 512)

Conforme a lo referido se comprende a la Normal Superior como un estrato de naturaleza antrópica, caracterizado por formas y sustancias heterogéneas y diversas, así como códigos y medios variados en su forma de organización. Esta estratificación, a partir de la Institución Normal, se expresa como creación de un mundo a partir del caos que requiere de un agenciamiento.

No obstante, hay que recordar, que "los agenciamientos no cesan de variar, de estar ellos mismos sometidos a transformaciones" (Deleuze y Guattari, 2004, p. 87). En esto radica la atinencia del concepto mismo, a tenor de esta propuesta. Solo al reconocer al agenciamiento en un régimen de signos ligados a la Normal Superior, en su multiplicidad, resulta posible considerar el constante devenir de la Normal Superior y sus transformaciones corporales e incorporales, en una inevitable mutabilidad de trasformaciones ontológicas, antropológicas, pedagógicas y normativas.

En virtud de lo expuesto, y siguiendo a Deleuze y Guattari (2004), es posible sacar algunas conclusiones generales en torno a la naturaleza de los agenciamientos y cómo estos inciden probablemente en las Instituciones Educativas Normal Superior:

Según un primer eje, horizontal, un agenciamiento incluye dos segmentos, uno de contenido, otro de expresión. Por un lado, es agenciamiento maquínico de cuerpos, de acciones y de pasiones, mezcla de cuerpos que actúan los unos sobre los otros; por otro, agenciamiento colectivo de enunciación, de actos y de enunciados, transformaciones incorporales que se atribuyen a los cuerpos. Pero, según un eje vertical orientado, el agenciamiento tiene por un lado partes territoriales o reterritorializadas, que lo estabilizan, y por otro, máximos de desterritorialización que lo arrastran. (p. 92)

Tales segmentos claramente se advierten a lo largo de este escrito acerca de un agenciamiento maquínico como colectivo que opera tanto en los contenidos pensados acerca de la 
formación desde lo instituido, como en las expresiones reconocidas e incorporadas de los actores implicados, sus cuerpos y pensamientos. Sin desconocer, claro está, las acciones, pasiones, modos de subjetivación y su vínculo con un territorio específico; aspectos estos que le confieren consistencia al agenciamiento mismo.

En este sentido, las/os maestras/os y las/os estudiantes, además de ser sujetos, son un acontecimiento que emerge en dicho proceso de agenciamiento formativo de la institución educativa Escuela Normal Superior. Agenciamiento que resignifica la relación maestra/odirectivos-estudiantes, potenciando la diversidad que habita entre ellas/os y su territorio, como una gran composición.

Por consiguiente, se trata de, a partir del agenciamiento de la formación de los maestros en la Normal Superior, reconocer las multiplicidades que emergen en este estrato y reconfigurar la naturaleza misma de la formación, a medida que aumentan las conexiones entre lo territorial y los sujetos no aislados, sino interconectados.

Conforme a lo anterior, desde esta apuesta de agenciamiento se intenta comprender e interpretar la voz de las Escuelas Normales Superiores de Santiago de Cali, reconocer el poder y saber que se encuentra en su naturaleza formadora, las posibilidades de desarrollo y crecimiento desde sus propias comprensiones, reconocimiento y empoderamiento como instituciones que forman maestras/os que llegan a lugares impensados, insospechados (zonas de alta vulnerabilidad social- urbano marginales rurales); lugares a los que otras/os profesionales de la educación no llegan (licenciadas/os, magister, doctoras/es).

Estas consideraciones en clave de agenciamiento potencian discursos y prácticas pertinentes al contexto en el que se proyecta la propuesta educativa de las Escuelas Normales Superiores de Santiago de Cali, al reconocer y resignificar los cambios legales que han tenido y el agenciamiento de la formación del maestro normalista, la estructura organizativa de ellas como instituciones educativas y su alcance formativo, entendiéndolo como aquello que las diferencia de otras instituciones educativas. Lo mencionado en este artículo finalmente pretende la identificación y construcción de un horizonte de sentido que les permita demostrar su legitimidad, su empoderamiento y asumir su mayoría de edad. 
Los lineamientos que a renglón seguido se comparten se afincan en la exhortación que se hace a las Escuelas Normales Superiores de Santiago de Cali, a cuestionar las normas como prescripciones que tienen que ser comprendidas desde lo que ellas establecen, para pensarlas, instalarlas y darles sentido en el contexto normalista, desde las comprensiones de sus actores. Siendo necesario, para esto, descentrar su tradición y moverse hacia otra, lo que en palabras de Mignolo (2007), como ya se señaló, significa situarse en "otro lugar de enunciación", en un "pensamiento fronterizo" o "pensamiento otro".

\section{PEDAGÓGICOS: FORMACIÓN SUPERIOR - COMPROMISO DE LOS ACTORES}

Se reclama desde este lugar el agenciamiento de la institución normalista, al reconocer y pensar a sus actores como sujetos protagónicos del proceso educativo; reconocer que están en capacidad de lograr una formación superior con niveles altos de calidad, en la medida en que se generen espacios de encuentro, más allá de las reuniones institucionales establecidas en las agendas y calendarios escolares; espacios de encuentro para discutir, comprender, acordar y determinar la formación de la/el maestra/o, sus posibilidades, sus retos y desafíos y sus implicaciones en el desempeño profesional y laboral de la/el formador de las/os formadores.

\section{HORIZONTE DE LA FORMACIÓN: IDENTIDAD DEL NORMALISTA}

Por su naturaleza y razón de ser, las Escuelas Normales Superiores en Colombia están comprometidas con la formación de la/el maestra/o para el ejercicio de la docencia en los niveles educativos de Preescolar y Educación Básica Primaria.

Es así como el acento de la formación requiere colocarse en las condiciones institucionales para potenciar el desarrollo de la identidad pedagógica, cultural, social, científica de la/el maestra/o. Lograrlo tiene complejidades y exigencias diversas y, en virtud de ello, es indispensable centrar la formación de la/el normalista en los conocimientos propios del saber de la/el maestra/o: Pedagogía - Didáctica - Investigación - Arte - Tecnología, en una relación teoríapráctica y práctica-teoría. Es necesario, también, pensar, organizar y desarrollar la Institución 
Normalista como campo y escenario de investigación y experimentación pedagógica. En la Institución Normalista se deben dar las condiciones para que directivos docentes, formadoras/es, formadas/os y egresadas/os desarrollen pensamiento pedagógico sobre problemas particulares de la formación de la/el maestra/o; un pensamiento pedagógico que genere saber que transforme las prácticas pedagógicas y formativas gestadas en ella, y que reconozca el saber pedagógico acumulado en la historia y trasegar de la Escuela Normal.

\section{DECOLONIZAR EL PENSAMIENTO Y ACTUACIÓN PEDAGÓGICA: TRADUCIR, INTERPRETAR Y COMPRENDER}

Para las Escuelas Normales Superiores de Santiago de Cali, esto significa desplazarse hacia el pensamiento de ellas y no centrarse exclusivamente en lo que la norma les traza. La invitación es a que se nieguen y se resistan a pensar y a actuar desde lo que otras/os disponen y ordenan, y decidan construir nuevos y diversos lugares desde donde soñar, pensar, enunciar y proponer nuevos conceptos, nuevas prácticas pedagógicas, nuevas formas de organizar y agenciar la formación de la/el maestra/o normalista.

Descolonizar el pensamiento y la actuación pedagógica demanda rupturas epistemológicas, toma de conciencia de sus problemáticas, de sus posibilidades, de sus proyecciones. Requiere, también, interpretar la realidad dentro de su contexto histórico y social, y comprender a los actores tal como son, sin intrusión alguna o contaminación de medidas formales o problemas preconcebidos, es decir, comprenderlos en el contexto de sus conexiones con la vida cultural y social. Es señalar la importancia y lugar de la/el maestro en el mundo del conocimiento $\mathrm{y}$, por ende, en los procesos de formación.

Descolonizar el pensamiento y actuación pedagógica significa reconocer la interculturalidad como destino que libera las voces encerradas bajo "las verdades" de los discursos hegemónicos de las normas. Consecuente con ello, exige el respeto de la diversidad y horizontalidad en el diálogo con otros actores escolares, con otras instituciones educativas, con otras Escuelas Normales Superiores y con otras instituciones de educación superior. 


\section{LINEAMIENTOS CURRICULARES: SUPERAR LAS VISIONES DISCIPLINARIAS}

Esto significa hacer rupturas, reconocer y transitar el aula como espacio que posibilita la construcción y expansión del conocimiento, la gesta de saberes, descubriendo que quien y quienes recorren este espacio traen consigo una cultura social cuyas réplicas toman auge en el reconocimiento y encuentro con el/los otro/s y lo otro. De esta manera, se reconoce que, en el aula elaborada $^{5}$, el acontecimiento pierde sentido, de ahí que se demande mirar la enseñanza no de forma instrumental, dogmática, uniforme si no como un lugar estratégico para compartir, generar y situar saberes sobre la misma realidad del sujeto en formación.

En este sentido, el/la formador/a de formadores/as no puede limitarse al conocimiento de los fundamentos de su disciplina, debe trascenderlos para conocer la estructura de la misma e identificar la relación con otros saberes. Reduciendo la fragmentación de la realidad y desvaneciendo las fronteras de su saber, es posible significar y dar sentidos a los procesos de formación. Romper fronteras, tejer redes y relaciones de conocimiento, reclama elegir caminos y rutas para que el concepto de interdisciplinariedad se constituya en

[un] concepto transversal que permite definir el lugar de la pedagogía y su relación con los saberes específicos, y demás componentes de la formación profesional de los docentes. Igualmente, propicia la elaboración de currículos que respondan adecuadamente al desarrollo de las competencias generales y específicas que requiere el docente en formación para constituirse en profesional en un determinado campo del conocimiento. (Rodríguez, 1999)

\section{ARTICULACIÓN ENTRE LA EDUCACIÓN BÁSICA, MEDIA Y EL PROGRAMA DE FORMACIÓN COMPLEMENTARIA}

La formación de la/el maestra/o en las Escuelas Normales Superiores de Santiago de Cali no puede quedarse atrapada en el Programa de Formación Complementaria y en la práctica

\footnotetext{
${ }^{5}$ La expresión "aula elaborada" es empleada para señalar la existencia de un escenario de enseñanza y aprendizaje preconcebido, planeado con rigidez, pensado y diseñado exclusivamente desde el interés institucional y del maestro; un escenario de enseñanza y aprendizaje que obedece a objetivos preestablecidos. En síntesis, es un aula que invisibiliza las expectativas, los intereses y las necesidades del estudiante en su contexto.
} 
pedagógica de las/os formadoras/es de formadoras/es. Debe y necesita ser un asunto transversal presente en todos y cada uno de los niveles ofertados por ellas. En este orden de ideas, la Pedagogía como uno de los principios pedagógicos que direccionan la propuesta de formación, precisa ser visibilizada en toda la estructura curricular y plan de estudios, colocándola en diálogo con todos los saberes que circulan en las áreas fundamentales del conocimiento, los proyectos pedagógicos obligatorios, los proyectos pedagógicos institucionales y en todo aquello que se propone para agenciar la formación del maestro. Lo que se requiere es transversalidad más allá de la estructura curricular de los niveles y de las áreas

\section{LA INVESTIGACIÓN FORMATIVA: EJE DE LA FORMACIÓN DEL MAESTRO}

Las Escuelas Normales Superiores de Santiago de Cali coinciden en definir la investigación como un proceso intelectual, posible bajo diferentes enfoques y modalidades, orientado a la generación de conocimientos. Reconocen que solo es posible desarrollar procesos de formación normalista de excelencia, en la medida en que maestras/os formadoras/es y en formación se vinculen a procesos de generación y asimilación crítica del conocimiento.

De igual manera, proponen la investigación, la innovación y el desarrollo tecnológico como las estrategias más adecuadas para el diálogo productivo entre los distintos saberes y disciplinas que circulan al interior del currículo y plan de estudios normalistas, en los distintos niveles, y del Programa de Formación Complementaria. Sin embargo, son claras en señalar que para transitar por estos caminos, se necesita algo más que voluntad pedagógica, identidad y compromiso institucional y que se hace necesario darle lugar a la investigación, evaluando y reconociendo las condiciones reales que la viabilicen.

\section{EL PRINCIPIO DE AUTONOMÍA PARA PENSAR LA FORMACIÓN, LA PEDAGOGÍA Y LA INVESTIGACIÓN COMO EJE DE TODA LA ACCIÓN INSTITUCIONAL}

En el contexto normalista, la formación es un proceso del sujeto; es decir, subjetivo, en el que la persona se transforma a sí misma de manera continua, logra la madurez de sus potenciales, 
alcanza un estilo de vida que le gratifica y contribuye a la construcción y desarrollo del mundo al que pertenece. Es un proceso permanente, continuo, incierto e individual en donde la/el maestro/a en formación se-piensa, se asume y toma decisiones sobre sí, sobre sus incertidumbres, sobre sus miedos, sobre sus posibilidades, sobre sus proyectos; y desde donde aprende a reconocer, valorar y asumir a la/el otro/a como un/a diferente válida/o, con la/el que crece.

De esta manera, las prácticas formativas y pedagógicas tienen sentido, significado y fundamento en la generación de caminos y rutas que viabilicen condiciones para que la/el normalista asuma su propia formación desde el conocimiento considerado en el currículo como válido, que merece ser enseñado a la/el maestra/o en formación y aprendido por ella/él, y en el reconocimiento del saber no escolar, del trabajo cooperativo, el diálogo, el trabajo integrado por proyectos y el reconocimiento de sus pares.

Lo que se requiere son prácticas formativas y pedagógicas que propicien experiencias que formen, que impacten el ser, el sentir, el pensar del maestro en formación como ser humano, como persona y en su opción de ser maestro en vínculo con sus experiencias de vida.

\section{REFLEXIÓN Y POSICIONAMIENTO SOBRE LA DOBLE CONDICIÓN: INSTITUCIÓN EDUCATIVA - ESCUELA NORMAL SUPERIOR}

La propuesta educativa de estas instituciones se desarrolla considerando una estructura curricular que necesita responder a procesos formativos instalados en lo que señala la Ley 115 de 1994 (El Congreso de la República, 1994) y el Decreto 1860 (MEN, 1994) del mismo año. Esta estructura curricular considera un plan de estudios organizado en áreas fundamentales y optativas, y para el Programa de Formación Complementaria, un plan de estudios en coherencia con lo que exigen sus propios marcos normativos vigentes y el decreto 4790 de 2008 (MEN, 2008), que lo organiza en Principios Pedagógicos y Ejes de Formación.

La organización de la jornada escolar y laboral de directivos docentes y docentes, reglamentada en el Decreto 1850 de 2002 (Presidencia de la República, 2002), no establece diferencia alguna en la organización escolar de los niveles de Preescolar, Básica y Media. El Programa de Formación Complementaria no interpreta las particularidades curriculares para el 
agenciamiento de la formación normalista, en coherencia con lo que señalan sus propósitos, sus intencionalidades y propuesta curricular contenidos en el plan de estudios previsto para tal fin.

El Programa de Formación Complementaria se caracteriza por poseer en su diseño, una estructura cimentada en principios de flexibilidad, contextualización y pertinencia, sin embargo, su movilización, ejecución y puesta en marcha no da cuenta de ello, dado que se queda atrapado en la lógica y rigidez organizativa de la Institución Educativa. En la práctica prevalecen las normas de la Educación Básica y Media, lo que limita el trabajo en comunidad académica de las/os formadoras/es de formadoras/es. También se ve limitada la investigación como medio que dinamiza el saber y la reflexión sistemática y documentada sobre la Pedagogía, la Práctica Pedagógica, la Investigación Formativa, la Didáctica y el Currículo, en tanto pretextos para la formación de la maestra y el maestro normalista.

La doble condición exige comprender e interpretar las normas en el contexto de una Escuela Normal que forma bachilleres con profundización en educación que pueden optar o no por ser maestras/os y también forma Normalista Superiores, y que en ambos procesos, el agenciamiento de la formación tiene que tener horizonte de sentido. Esto significa pensar y proponer otras formas de organización desde sus reflexiones y reconocimiento de sus potenciales en términos del aprovechamiento del talento humano presente en la planta de cargos, es decir, dar oportunidad a otros liderazgos, independientemente del cargo que se tenga dentro de la organización.

\section{RECONOCER Y POTENCIAR EL TALENTO HUMANO: RE-SIGNIFICAR LAS FUNCIONES}

La articulación curricular entre niveles, grados y el Programa de Formación necesita pasar de los discursos a la práctica, lo que implica pensar la necesidad de establecer un diálogo directo y recíproco entre lo administrativo y lo pedagógico que, si bien son procesos con responsabilidades propias y particulares, no signifique el sometimiento de lo uno sobre lo otro, sino trabajo mancomunado y solidario. 
Un diálogo directo y recíproco entre lo administrativo y lo pedagógico pasa también por la resignificación del concepto de directivo docente ${ }^{6} \mathrm{y}$ de las funciones que por normativa señala el Estado. Las coordinaciones son llamadas a pensar lo pedagógico, lo formativo y la convivencia de una manera integrada que considere al/la normalista como ser humano integral y multidimensional. En este sentido, la organización escolar no debe separar lo académico pedagógico formativo de los asuntos de convivencia para, así, centrar la formación en el sujeto, como un proceso personal, social y significativo dentro del cual interioriza, apropia y acumula conocimientos y experiencias que le permiten buscar y construir, con el apoyo de otras personas, las condiciones para que todos los conocimientos y experiencias se enriquezcan y se transformen en su proyecto de vida.

Al Programa de Formación Complementaria, el Estado le exige dar cuenta de procesos y productos en el ámbito de la práctica pedagógica del normalista, la investigación formativa y la proyección y extensión a la comunidad. Este ejercicio demanda una revisión de las funciones docentes, más allá del trabajo del aula y de lo que establece el Decreto 1850 de 2002 (Presidencia de la República, 2002), que expresa de manera categórica que la/el docente cumple funciones estrictamente de docencia y dentro del aula. Este mandato imposibilita destinar tiempos para la investigación, para la extensión y para el diseño de propuestas pedagógicas de largo alcance que lean los tiempos y que planteen otras miradas sobre lo educativo y lo formativo; tiempos para pensar la academia, la Institución Educativa y la Escuela Normal. Urge pensar las funciones docentes de cara a la investigación, la proyección y la extensión, en un todo integrado.

\section{AMPLIAR EL HORIZONTE FORMATIVO: APERTURA A OTROS MUNDOS Y A OTRAS EXPERIENCIAS}

La apertura de las Escuelas Normales al mundo exterior, a otros mundos, a otras experiencias, es acercarse al conocimiento y la experiencia de otras/os, a la vida académica y de otras organizaciones, es oportunidad para aprender de otras/os y con otras/os. Es romper los límites, las fronteras de la Escuela Normal como institución, ruptura que busca ampliar el panorama de lo pedagógico, de las problemáticas de la formación, de las prácticas pedagógicas, de los contextos

\footnotetext{
${ }^{6}$ En el contexto de la investigación, “directivo docente” se refiere a los cargos de rectoría y coordinaciones.
} 
sociales y culturales. De esta manera, el establecimiento de alianzas estratégicas a través de convenios de cooperación académica interinstitucional se convierte en oportunidades y medios que hacen posible la apertura a otros mundos y a otros modos de formar.

\section{CONCLUSIONES}

Las conclusiones que se establecieron a la luz de los hallazgos permiten afirmar que los cambios legales de las Escuelas Normales Superiores de Santiago de Cali han tenido comprensiones y significados diversos y múltiples, conectados directamente con el agenciamiento de la formación normalista, sus conceptos, sus prácticas, el currículo y plan de estudios, y la organización institucional.

Por un lado, lo externo, lo mandado, lo ordenado desde estamentos gubernamentales nacionales y organismos internacionales se refleja, de cierto modo, al interior de la Escuela Normal como Institución Educativa, pero, por otro lado, se pudo también constatar cómo en el contexto vital de las Escuelas Normales Superiores, la/s voz/voces de los actores permanecen ausentes frente a lo prescriptivo, toda vez que las vicisitudes que configuran la ontología de las Normales Superiores hacen que estas se encuentren en una incertidumbre normativa, como se expone en este artículo.

Se percibieron significados profundos y desconocidos en relación con el problema de la formación y el quehacer de la/el maestro normalista. Las voces de los actores, sus discusiones, sus propuestas, sus sueños y sus ideales de formación, se quedan atrapados en discursos bien logrados, que no son reconocidos como válidos, viables y posibles, ni llegan a ser instituidos en las prácticas formativas, dado que la norma establece unos márgenes operativos que las subyugan, las someten; a razón de ello, "lo superior", como lo señalan las normas jurídicas, es solo declaración, mas no acción. 


\section{LAS CONCEPCIONES Y LAS PRÁCTICAS}

El reconocimiento de la práctica pedagógica como un elemento clave y transversal en la formación de la/el maestro normalista fue uno de los cambios más significativos dados en las Escuelas Normalistas Superiores. El cambio se dio en lo conceptual y en lo metodológico.

A nivel conceptual, se transitó de lo instrumental, instruccional y rutinario, a una práctica pedagógica pensada, problematizada, reflexionada, que genera saber pedagógico desde la vivencia de la experiencia tanto de formadoras/es de formadoras/es como de la/el normalista en

formación. Se transitó a una práctica pedagógica investigativa que se asume como "eje transversal de la formación", que integra y articula lo pedagógico y lo investigativo, concibiendo lo pedagógico como el saber fundante del maestro que le confiere identidad profesional y le posibilita una comprensión critica de los procesos pedagógicos que tienen lugar en el aula y en la escuela y lo investigativo, como un proceso inherente al trabajo pedagógico del maestro, que le posibilita el desarrollo de reflexiones sobre la práctica, en la práctica y para la práctica, reflexiones que se constituyen en saber pedagógico.

En cuanto a lo metodológico, la práctica pedagógica exigió discusiones, reflexiones y propuestas, que llevaran a la/el normalista en formación a plantearse problemas pertinentes a la realidad que vive en los distintos campos de práctica; asumir con responsabilidad y con independencia la reflexión sobre el quehacer pedagógico; y comprometerse en el diseño y estructuración de proyectos investigativos articulados a los espacios académicos propuestos en el plan de estudios en cada uno de los semestres.

\section{CURRÍCULUM Y PLAN DE ESTUDIOS}

Los cambios dados en el currículum y plan de estudios de las Escuelas Superiores Normalistas crearon inquietudes y necesidades nuevas de conocimiento, de indagación y de preguntas en relación con el proceso formativo de la/el normalista, considerando otros asuntos, más allá del desarrollo de los contenidos programáticos de los espacios académicos colocados en el plan de estudios. Estos cambios posibilitaron pasar de la asimilación de un conocimiento elaborado y preparado por otros a comprender el conocimiento como el resultado de la recuperación de la 
experiencia y de los saberes previos de la/el normalista en formación. Se trata de incorporar a estos los nuevos conocimientos, para que juntos den lugar a un conocimiento nuevo, construido socialmente.

Se ha avanzado hacia un currículo y un plan de estudios que prioriza la investigación, entendida como la formación de una actitud investigativa que implica una reflexión sobre la propia realidad del sujeto, sobre el compromiso con la propia formación y sobre el rol social de la/el maestra/o normalista como factor altamente influyente en la formación de otras subjetividades, lo que exige una postura crítica frente a la concepción de desarrollo humano, la sociedad y la cultura en la cual interactúa el sujeto hoy.

Pese a lo expuesto, es necesario pensar el desarrollo del currículum y del plan de estudios dentro de una concepción de horarios académicos más allá de la organización centrada en la rigidez de la hora clase que establece tiempos precisos e inflexibles para el desarrollo de las disciplinas en cada semestre. Hay que pensar su desarrollo desde una perspectiva y mirada de integración interdisciplinar entre espacios académicos, movilizándolos a la luz del planteamiento de preguntas problema que encuentran su origen en la reflexión crítica sobre los contenidos de enseñanza propuestos por cada saber disciplinar, en la experiencia de práctica pedagógica y en la experiencia de formación en investigación. Es clave proponer y desarrollar contenidos de enseñanza desde una perspectiva intercultural, lo que implica situarse en la realidad del contexto en el que se desarrolla el Programa de Formación Complementaria, a la vez que brindar a los maestros en formación la oportunidad de reconocer, valorar y considerar sus conocimientos previos y sus pensamientos y creencias sobre la enseñanza.

\section{DECOLONIZAR EL PODER PRESENTE EN LA ESTRUCTURA ORGANIZACIONAL}

La organización unidireccional de las IEENS (Institución Educativa Escuela Normal Superior), hacen que el Programa de Formación Complementaria sea un agregado de la formación, un apéndice de la institución, y sea visto como algo extraño y desconocido. La organización está centrada en el cumplimiento de la norma a razón del manejo de los tiempos escolares, asignación de responsabilidades académicas a docentes, funciones de directivo docentes ausentes y aisladas 
de los propósitos de formación de la/el maestra/o normalista, ancladas, estacionadas en el cumplimiento de lo que establecen los manuales de funciones.

Es urgente avanzar hacia una organización institucional "multidireccional” que dé lugar al pensamiento pedagógico y la acción pedagógica de las/os formadoras/es de formadoras/es, en tanto sean vistos como actores capaces de diseñar propuestas que impacten la formación de la/el maestra/o normalista, en diálogo con las exigencias del Estado y por encima de ellas, es decir, agenciar la formación de la/el maestra/o normalista desde el reconocimiento de sus posibilidades de desarrollo en el contexto cultural, político y social en que ejercerá la docencia.

Se requiere una organización institucional que dé lugar y sentido a lo múltiple, a la diferencia, a la emergencia de lo particular de los colectivos sociales y que sea capaz de experimentar, de impulsar nuevas experiencias, nuevas reflexiones, nuevas prácticas: una IEENS multidireccional que se piensa y asume como escenario de cultura que contribuye a su producción, reproducción, recreación y transformación; que comprenda y asuma la autonomía como el reconocimiento, que desde el marco normativo, se hace de su capacidad para determinar su propio rumbo, definir concepciones, procesos y líneas de acción que orienten la vida y dinámica institucional, y las acciones formativas que en ella se piensan, diseñan y desarrollan.

Es importante que se piense y determine las funciones propias de las coordinaciones más allá de su denominación "convivencia- académica" para que estas se asuman desde una concepción holista y de integralidad formativa coherente con la propuesta pedagógica contenida en el Proyecto Educativo Normalista (PEN). Hay que pensar las coordinaciones desde lo Académico - Investigativo- Práctica Pedagógica y Proyección y Extensión, y asimismo las funciones docentes. Se requiere una organización que supere la homogeneidad y de lugar a lo heterogéneo como criterio de formación, y a significar y resignificar la diferencia y la particularidad; que reconozca la necesidad de plantear y desarrollar proyectos y programas, en diálogo con expectativas, intereses y requerimientos de la sociedad.

La formación de la/el maestra/o normalista requiere de decisiones y acciones organizativas que generen una cultura que favorezca la participación de las/os formadoras/es de formadoras/es en los procesos de cambio institucional, en lo concerniente a la incorporación de enfoques pedagógicos que se alimenten de tendencias, discursos y prácticas pedagógicas, 
filosóficas y sociológicas hoy en discusión. Se requiere una organización normalista que piense y asuma el cumplimiento de la norma en un contexto de libertad y de autonomía que potencia la construcción particular o colectiva de saberes que ayudan a solucionar problemas, que atienden las necesidades, y los requerimientos o demandas de otros grupos poblacionales.

Las instituciones educativas, esto es las Escuelas Normales Superiores, tienen la urgencia de pensar y diseñar estrategias de formación en coherencia con las características de los contextos territoriales, los modelos pedagógicos, las políticas públicas y las demandas del mundo globalizado, que contribuyan, desde la equidad y la convivencia pacífica, al aseguramiento del bien público, al establecimiento de una sociedad plural abierta interculturalmente a la transformación de la realidad Caleña, Vallecaucana y Colombiana. Deben proponer una organización de cara a la Formación Superior para autosignificarse, autodefinirse, autogobernarse y autoafirmarse para pensar la formación y el desarrollo humano de la/el maestra/o, a la luz de las relaciones de sentido que necesitan tejerse, entre autonomía, marcos normativos y su agenciamiento en la formación de la/el maestra/o normalista.

El reto más significativo y relevante que se plantea a las Escuelas Normales Superiores de Cali es el de dejar atrás una organización vertical, jerárquica y rígida, centralizada y direccionada en lo ordenado por las normas que las regulan, en el que todo conduce a concebir la formación de la/el maestra/o normalista como una actividad prescrita, definida, regulada, instrumentada, controlada y evaluada por el Estado, para proponer y lograr una organización institucional que, en el marco de lo normativo, favorezca experiencias formativas que potencien y propicien en la/el normalista, pensamiento crítico y propositivo, ético profesional y el desarrollo de su proyecto de vida en condiciones de calidad.

\section{A MANERA DE CIERRE}

En el contexto de la investigación "Agenciamiento de los cambios legales en la formación de los maestros normalistas de Santiago de Cali - Un estudio comprensivo - (2004-2016)" se invita a las Escuelas Normales Superiores de Santiago de Cali a que desplacen el lugar de la enunciación hacia el pensamiento de ellas mismas; se nieguen y se resistan a pensar y a actuar desde lo que 
otros disponen y ordenan, y decidan construir nuevos y diversos lugares desde donde soñar, pensar, enunciar y proponer nuevos conceptos, nuevas prácticas pedagógicas, nuevas formas de organizar y agenciar la formación de la/el maestro normalista. Lo expresado les demanda rupturas epistemológicas, toma de conciencia de sus problemáticas, de sus posibilidades, de sus proyecciones.

\section{REFERENCIAS}

MEN. (s.f.). Políticas y sistema Colombiano de Formación y Desarrollo Profesional Docente. From mineducacion.gov.co/: https://www.mineducacion.gov.co/cvn/1665/articles208603_archivo_pdf.pdf

Rodríguez, M. E. (1999). La interdisciplinariedad en la formación docente. Temas de Acreditación. (1), 44.

El Congreso de la República. (1994). Ley 115 de febrero 8 de 1994. Por la cual se expide la ley general de educación. Bogotá: El Congreso.

MEN. (1994). Decreto 1860 de 1994. Por el cual se reglamenta parcialmente la Ley 115 de 1994, en los aspectos pedagógicos y organizativos generales. Bogotá: Diario Oficial No 41.473 .

MEN. (2008). Decreto 4790 de 2008. Por el cual se establecen las condiciones básicas de calidad del programa de formación complementaria de las escuelas nonnales superiores y se dictan otras disposiciones. Bogotá: Ministerio de Educación Nacional.

Presidencia de la República. (2002). Decreto 1850 de 2002. por el cual se reglamenta la organización de la jornada escolar y la jornada laboral de directivos docentes y docentes de los establecimientos educativos estatales. Bogotá: Diario Oficial No. 44.901 jueves 15 de agosto de 2002. 
Congreso de la República, C. (1994). Ley 115 de Febrero 8 de 1994. Bogotá: Congreso de la República de Colombia.

M.E.N. (2013). Sistema Colombiano de Formación de Educadores y Lineamientos de Politica. Bogotá: M.E.N.

Peña, F. B. (1996 ). Praxis y Poiesis en la pedagogía y la didáctica. Lúdica pedagógica , 11-16.

El Congreso de la República. (2001). Ley 715 de 2001. Por la cual se dictan normas orgánicas en materia de recursos y competencias. Bogotá: El Congreso.

Pineda, L. M. (2015). La práctica pedagógica investigativa del maestro formador en las escuelas normales superiores de Cali: Concepciones epistemológicas e impacto en la formación del maestro normalista. Recuperado de https://goo.gl/PYaELg

Ríos, A. G.(2004). Investigación y formación de docentes. Plumilla Educativa, (3), 153-158. 